Voix et Images

volxetimages

\title{
L'écriture iconique. Sur deux « Mirors » de Roland Giguère
}

\section{Roger Chamberland}

Volume 9, numéro 2, hiver 1984

Roland Giguère

URI : https://id.erudit.org/iderudit/200438ar

DOI : https://doi.org/10.7202/200438ar

Aller au sommaire du numéro

Éditeur(s)

Université du Québec à Montréal

ISSN

0318-9201 (imprimé)

1705-933X (numérique)

Découvrir la revue

Citer cet article

Chamberland, R. (1984). L'écriture iconique. Sur deux « Mirors » de Roland

Giguère. Voix et Images, 9(2), 47-58. https://doi.org/10.7202/200438ar d'utilisation que vous pouvez consulter en ligne.

https://apropos.erudit.org/fr/usagers/politique-dutilisation/ 


\title{
L'écriture iconique. Sur deux "Mirors" de Roland Giguère.
}

\author{
par Roger Chamberland, Université Laval
}

\begin{abstract}
"Alles Wissen Entsteht durch separation, abgrenzung, Beschränkung; kein absolutes wissen eines Ganzen".

"Tout savoir naît de la séparation, de la délimitation, de la restric. tion; aucun savoir absolu d'un tout».
\end{abstract}

\section{NIETZSCHE}

Miror. Qui est Miror? Le lisible, celui des XXXI strophes auxquelles il prête son nom, ou les visibles, double travail graphique dont les résultats, pour peu qu'ils s'affranchissent du lisible premier, acquièrent une spécificité par laquelle le statut de la figure est décisif. Une première version insérée dans le recueil le Défaut des ruines est d'avoir des habitants (1959), 1 la seconde parue dans l'album de dessins $\dot{A}$ l'orée de l'oeil (1981). ${ }^{2}$ De fait, le personnage de Miror occupe une place privilégiée dans l'oeuvre, tant littéraire que graphique, de Roland Giguère, puisque sa récurrence atteste d'une catharsis, spéculum imaginal qui en approfondit sans cesse la signifiance. Délaissant le texte strictement littéraire, celui des XXXI strophes qui enchâssent l'illustration avec le texte que nous avons retenu, nous essaierons de mieux cerner le rapport existant entre les deux tracés, le dessin et l'écrit périphérique (l'ergon kantien) - soit le titre ou ce qui en tient lieu -, et de procéder à une analyse du champ visuel des deux dessins, où s'investit le rapport performatif des unités signifiantes mises en contact.

Roland Giguère est l'un des premiers artistes écrivains du Québec à s'être manifesté autant par l'écriture que par les arts visuels. Depuis plus de

1. Le Défart des ruines est d'avoir dles habilants, Montréal, Éditions Erta, 1957, 107 p. (v.pp. 13-39 et plus spécialement les pages 26-27). «Miror» a été reproduit, sans dessin, dans la Main all fell, 1949-1958, Montréal, Éditions de l'Hexagone, 1973, pp. 12-29. Le dessin fut reproduit dans "Connaissance de Giguère", la Barre du jour, numèros 11-12-13, décembre 1967, 197 p. (v.p. 72).

2. Ál'orée de l'oeil, Saint-Lambert, Éditions du Noroît, 1981, 109 p. (v.p. 61). 
trente ans, ces deux productions concomitantes, aussi importantes l'une que l'autre, se sont effectuées jusqu'à ce que l'un des procédés supplante l'autre et se le rende complémentaire. $\mathrm{Si}$, au début, la dimension littéraire a occupé la majeure partie de l'espace du papier, petit à petit elle cède le terrain à des éléments graphiques qui prennent forme et se multiplient à l'infini:

Je commençai, vers les années 52-53, par dessiner dans les marges de mes poèmes. Petit à petit, mes dessins réclamèrent de plus grandes marges, envahirent la page, ne tolérant parfois qu'une phrase ou deux, quelques mots, et finirent par chasser complètement les mots pour occuper, en maîtres incontestés, la feuille entière. ${ }^{3}$

Ainsi, devons-nous prendre la figurabilité comme étant l'opération par laquelle une idée abstraite des pensées du rêve est remplacée par une figure visible où se réalise une altération au niveau de l'expression. Ce changement d'expression est libidinal, selon Freud, puisque le continuum expressif, le dessin, relève d'une énergétique des pulsions parce que la figure interagit avec le texte grâce au déplacement et à la condensation qui collaborent ensemble pour former un espace surdéterminé.

\section{Le texte mis en représentation}

Dès 1953, dans la partie du recueil le Défaut des ruines est d'avoir des habitants, intitulée «Miror», Giguère dessine à l'encre un «Portrait de Miror» (mais qui ne portera ce titre qu'en 1968, lorsque la Barre du jour, lui consacrant alors un numéro spécial, reproduira ce dessin avec l'indication «Portrait de Miror» et le texte original) auquel se jouxtent cinq petits vers imprimés en regard du dessin (cf. figure 1). Sans effort de concertation, nous déduisons que la créature esquissée à grands coups de plumes se nomme Miror puisqu'elle se trouve dans une section du recueil où l'on parle d'un être mi-homme, mi-créature fictive. Le court texte qui l'accompagne tenant lieu de titre (une telle pratique est fréquente chez Giguère), en spécifie l'authenticité. Le message linguistique, la description partielle dénotée de l'image, équivaut à un ancrage, au sens où l'entend Roland Barthes, ${ }^{4}$ de certains denotata. Or, ce qui est moins évident dans le dessin de Giguère, ce sont précisément les dénotateurs correspondants, dans la mesure où ceux-ci sont désignés. Il y a en quelque sorte une transgression énergétique si l'on considère que la figuration fait correspondre un «équivalent» plastique au discours: la représentation. Équivalence parce que l'image se fait reconnaître comme étant le référent dont parle le texte. Le personnage tenu à distance dans l'espace du discours se trouve «présenté», montré dans la forme visible. La ratio autorise le transfert du

3. Giguère, Roland, Forêt vierge folle, Montréal, Éditions de l'Hexagone, 1978, p. 109.

4. Barthes, Roland, "Rhétorique de l'image», Communications no 4, 1964, p. 45. 


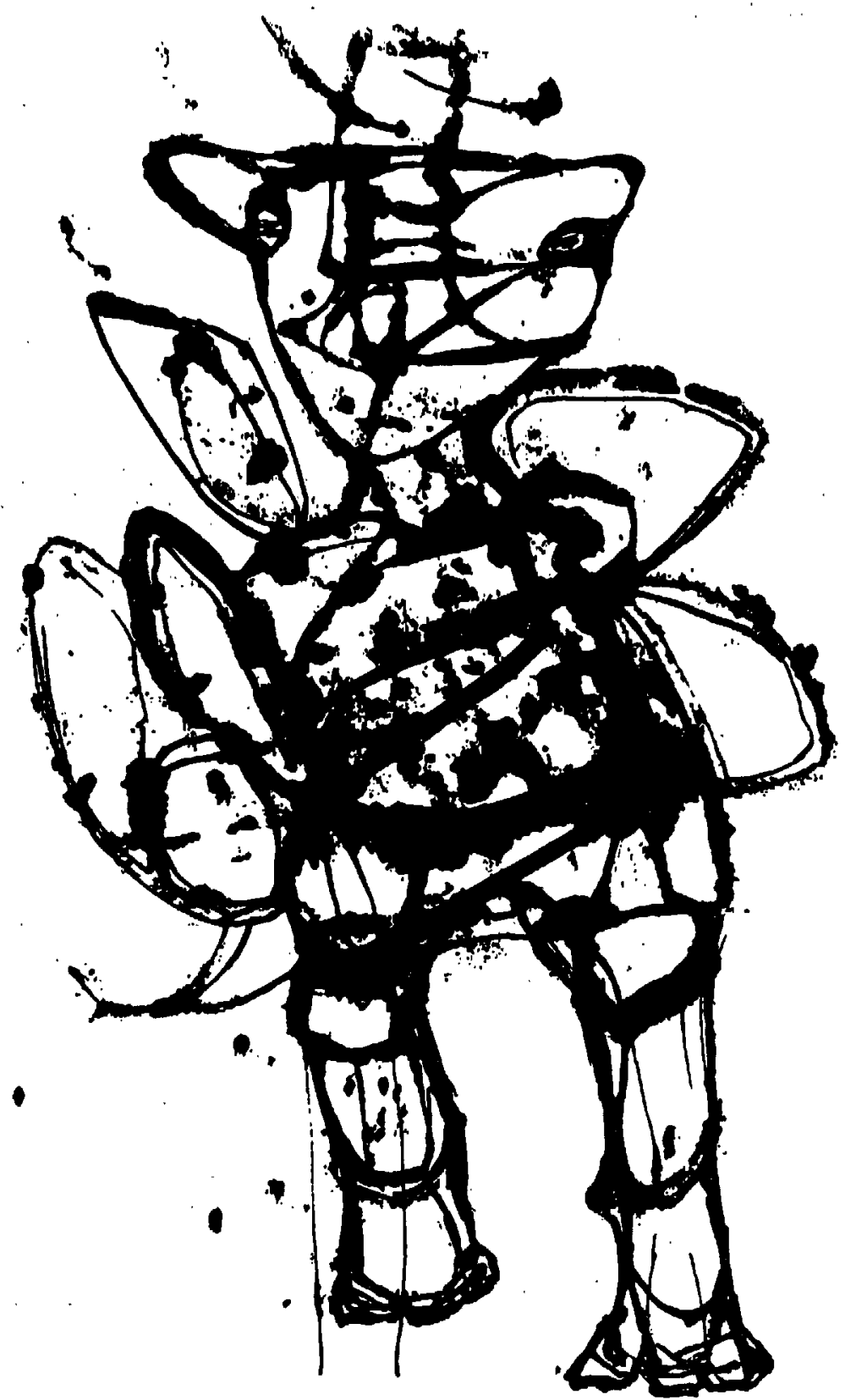

PORTRAIT DI: MIROR (encre de R.(i., 9-7-53).

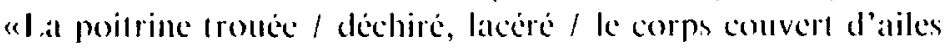
inulikes / il n'ira pats loin / all sortir de son domaninen R. (i.

Figure I: "Portrait de Miror", ex/rait de ke Défaut des ruines est d'avoir des habitants, reproduit dans la Barre du jour, décembre 1967 - mai 1968. 
texte à la figure, en tant que mode spécifique de la faculté de substitution. En exhibant son pôle référentiel, on offre un substitut visible au signe linguistique.

Le dessin a toujours été pour moi une expression aussi naturelle que l'écriture; le dessin est une écriture et c'est sans doute pourquoi j'y attache une si grande importance. Il y a dans le dessin un ton confidentiel, regarder les dessins d'un peintre c'est un peu feuilleter son journal personnel. ... Le dessin lui, se présente à nous dans toute sa nudité, sans parure, la ligne seule donne forme, le noir est toute sa gamme, sa pauvreté fait sa force. 5

Le lecteur qui lit «la poitrine trouée / déchiré lacéré / le corps couvert d'ailes inutiles...», possède les précisions référentielles qui favorisent la reconnaissance au niveau du dessin proprement dit. Privé du texte écrit, le dessin perd son pouvoir représentatif, ou du moins la portée en est atténuée et il n'assume plus que la fonction de la figuration. (Encore faut-il considérer qu'il est intégré à un ensemble beaucoup plus large, très autoréférentiel, qui en décrit certains aspects.) Aussi n'est-il pas rare de voir un dessin, un tableau ou un ensemble d'oeuvres et d'y lire un texte écrit plus ou moins long, allant du titre bref au catalogue d'exposition: "Toute notre expérience de la peinture comporte en fait une considérable partie verbale. Nous ne voyons jamais les tableaux seuls, notre vision n'est jamais pure vision». 6 Ce recours au texte est d'autant plus prégnant qu'il y a une lexicalisation immédiate et nécessaire de la représentation dans la mesure où le discours se tient à l'articulation du lisible et du visible. Déjà, Freud dans Métapsychologie avait remarqué ce phénomène de concaténation dans la représentation d'objet consciente qui se scinde en représentation de mots et en représentation de choses. Le visible et le lisible se nouent ainsi d'un à l'autre et coexistent dans l'oeuvre puis, successivement, dans la saisie du récepteur. "Le tableau est un texte figuratif et un système de lecture»" 7 plutôt des parcours de sens qui ne tendent pas à découvrir les signifiés mais à constituer les signifiants puisqu'il y a élimination du référé, analogon du monde; l'objet pictural se désignant lui-même.

Dans le dessin «Miror», il n'y a aucun désigné possible, ne serait-ce que par certains traits anthropomorphiques et zoomorphiques qui, tout en suggérant très fortement une adéquation avec le texte "Miror», en sont fort dissemblables. Néanmoins, il existe entre le texte et l'image une fonction de dénomination qu'il est nécessaire d'envisager. Le dessin de Roland Giguère délimite dans un espace donné, la feuille, quelques traits graphiques qui marquent les limites d'un dedans et d'un dehors. Ces lignes continues et élémentaires, parfois entrelacées ou densifiées pour marquer l'opacité d'un

5. Giguère, Roland, op. cit., p. 102.

6. Butor, Michel, les Mots dans la peinture, Paris et Genève, Flammarion et Éditions Albert Skira, coll. Champs, 1980, p. 5.

7. Marin, Louis, Éludes sémiologiques, Paris, Éditions Klincksieck, 1971, p. 19. 
dedans, ne figurent pas en soi de propriétés attribuables en propre au Miror des cinq lignes dénominatives, même si, comme nous le verrons plus loin, il est possible d'y déceler quelques éléments de duplication.

La réplicabilité est agissante conformément à une convention graphique qui «permet de transformer sur le papier les éléments schématiques d'une convention perceptive ou conceptuelle qui a motivé le signe». 8 Ces conventions régissent les modes de production des fonctions sémiotiques où les fonctifs sont créés par le dessin plutôt que l'inverse. Toutes formes de transformations iconiques sont elles-mêmes réductibles a l'activité d'institution de code $-U$. Eco les a classifiés selon une dizaine de possibilités: codes perceptifs, de reconnaissance, de transmission, tonaux, iconiques, iconographiques, du goût et de la sensibilité, rhétoriques, stylistiques, de l'inconscient. ${ }^{9}$ Le lecteur, connaissant ces conventions, peut procéder au décodage, à cause précisément de ces stimulus de remplacement qui, dans cette représentation relativement conventionnelle, favorise la signification ou du moins sa reconnaissance visuelle.

Dans le continuum expressif, il est possible de reconnaître des unités de contenu qui confirment la relation d'ancrage des deux textes, le linguistique et l'iconique. «La poitrine trouée» se visualise par ces taches noires, à hauteur de tronc, et suggère une profondeur du corps, son épaisseur (la troisième dimension) que le dessin ne peut rendre adéquatement que par un artifice tel la perspective qui n'existe pas dans la réalité mais que la culture nous a appris à reconnaître. «Déchiré lacéré», par son masculin ne renvoie plus à la poitrine mais à un sujet absent linguistiquement mais désigné iconiquement dans les signes iconiques qui sont ajointés au texte. Ces marques de blessures corporelles sont, à l'instar des trous de la poitrine, signifiées par ces traits noirs plus ou moins denses qui traversent de part en part le visage, le tronc et les jambes de Miror. - Nous utilisons cette dénomination anatomique par pure codification culturelle. - Les configurations latérales qui surgissent là où des ailes sont susceptibles d'y être, actualisent «le corps couvert d'ailes inutiles").

Le passage du type à l'occurrence s'effectue selon une ratio difficilis, c'est-à-dire qu'il y a concordance entre l'occurrence expressive et son propre contenu en vertu d'une institutionnalisation du système de l'expression tel qu'il a été prévu par le code et que vient renforcer le message linguistique. Dans le dessin, les traits pertinents des ailes (latéralité, morphologie), par exemple, sont reproduits de façon rudimentaire et imprécise, simulée, mais cela est suffisant puisque leur présence en un tel endroit a été enregistrée par la culture. - On peut facilement envisager qu'il eût été plus plausible pour la culture helléniste de placer ces ailes aux chevilles de Miror.

8. Eco, Umberto, «Pour une reformulation du concept de signe iconique», Communications no 29,1978 , p. 152

9. Eco, Umberto, «Sémiologie des ṃessages visuels», Communications no 15, 1970, pp. 39-40. 
Finalement, les deux dernières lignes, «il n'ira pas loin / au sortir de son domaine», caractérisent le mouvement du pied levé (le gauche) qui, dans l'instant présent, se déploiera pour se poser au sol et franchir un espace donné. L'écrit anticipe une conclusion dont le dessin ne manifeste aucunement la virtualité. (Que savons-nous de son domaine? comment savoir s'il se rendra à destination?). Tout au plus, ce fragment textuel rend-il plus probante, en l'accentuant, l'illusion de mouvement que suggère la jambe fléchie, prête à faire un pas. D'ailleurs, les deux traits continus, non fermés, parallèles à l'autre jambe, représentent en quelque sorte la position initiale de la jambe qui, ayant rompu son immobilité première, en acquiert une seconde, quelque peu déplacée et en suspens par rapport au sol. De plus, le décentrement du torse et de la tête par rapport aux jambes crée un effet de déséquilibre, concomitant au geste de se mouvoir et qui en suractive la valeur. Le pied droit posé au sol, sur une surface absente, non-délimitée, le pied gauche légèrement surélevé, Miror est immobilisé dans cette posture inconfortable, prêt à actualiser la prescription du texte. Ce même membre, dessiné en raccourci, avec des plis à deux articulations, rend opérante la notion de perspective. Dès lors, un troisième axe compositionnel s'ajoute aux deux premiers et donne une masse (présupposée) au personnage. De plus, il est à noter que le dessin marque un temps d'arrêt dans le mouvement déjà engagé de Miror. D'où l'on peut déduire que le personnage est doté de mobilité, qui résout la dialectique animé / inanimé, et se meut grâce à ce système inusité à trois articulations. Son geste freiné en plein élan, et esquissé à grands traits, rend plausible l'énoncé de la strophe sans toutefois la confirmer.

En aucun cas, on ne peut affirmer que le texte verbal «interprète» littéralement le texte pictural et vice-versa. Tout au plus parvenons-nous à insérer texte et dessin, présentés en hors-texte, comme une strophe supplémentaire aux trente et une autres qui tentent de définir Miror. L'expression picturale est édifiée sur la base d'un modèle de contenu dont l'existence est corrélative à sa propre manifestation, tandis que le message verbal nous retourne à l'analyse de ses signes linguistiques. La coïncidence de ces deux messages est d'autant plus forte qu'elle s'appuie sur une série de signaux denses dont la principale caractéristique consiste à reproduire au maximum les conditions de la perception.

De fait, la naissance de l'un et l'autre textes s'est effectuée selon la même instance compositionnelle; l'artiste ayant comme matériau commun à ces deux types d'activités, la plume, l'encre et le papier puisque, au dire même de Giguère, dessin et poésie se bousculent à la pointe de l'objet traceur. Mais comment s'articule cette double émergence? «Est-ce qu'il y a un rapport logico-sémantique entre la légende et l'image? Ou bien est-ce qu'il y a un rapport polylogique?» Tout le rapport entre le visible et le lisible tient dans cette question que posait Roland Barthes il y a quelques années. ${ }^{10}$

10. Barthes, Roland dans, «La peinture et l'écriture des signes», Coloquio (artes), 2e série, no 20, (décembre 1979); p. 29. 
La scénographie sommaire que nous venons d'effectuer nous permet de fournir quelques éléments de réponse. Nous constatons que Roland Giguère n'a retenu que quelques traits pertinents de son esquisse, au détriment de plusieurs autres (triangularité du visage, larachycépalité, périssodactylité, triple articularité, ...); c'est dire qu'un premier clivage s'effectue dans un système de lexies ${ }^{11}$ et met en évidence le déplacement métonymique qui s'opère dans le rapport entre le texte et la figure qui constituent l'ensemble "Miror». Le dessin, quant à lui, commande une lecture de sens qui multiplient l'image et déterminent «... un complexe d'unités lexicologiques coextensives à la surface et la définissant comme un niveau de signifiant».12 C'est le nombre et le type de lectures qui définissent le dessin car la lexie permet de découvrir des signifiants et, de surcroît, d'isoler une matrice. Nommons-la, avec Jean-François Lyotard, matrice figurale: «connivence radicale de la figure et du désir (...) qui articule fortement l'ordre du désir et l'ordre du figural par la catégorie de transgression". ${ }^{13}$ C'est à ce niveau de la figurabilité que le travail du rêve agit sur la figure, qu'elle soit verbale ou iconique. L'épreuve de la scotomisation devient opératoire et agit sur l'efficience du continuum expressif, au plan de la valeur de significations, car il prédomine sur le texte verbal dans la mesure où ce dernier, acquérant une forte valeur en terme d'indices, structure une lexie, par définition toujours incomplète.

Celui dont on parle dans le texte, et qui en est forclos, adopte sous le ductus giguérien une morphologie visuelle: ce en quoi elle se présente comme une «nébuleuse de contenu» ${ }^{14}$ puisqu'elle ne possède que des portions de contenu.

Cette double configuration - texte verbal et texte pictural - est une réflexion spéculaire où les deux textes se renvoient à l'épaisseur de leur signifié, ad infinitum. Le sujet du dessin n'a d'existence structurale que dans sa textualité. Il doit être lu, à partir de la thésaurisation du sens, afin que soit reconstituée une surface idéale du dessin: un plan dénoté.

\section{Sur un titre et son icône}

L'année suivant la publication de le Défaut des ruines est d'avoir des habitants, Roland Giguère effectue un second «portrait» de Miror, intitulé comme tel dans le coin inférieur gauche. Cette deuxième version de Miror ne sera connue qu'en 1981, lors de l'exposition rétrospective des dessins de Giguère tenue à la Galerie Treize (Montréal) et reproduite dans l'album $\grave{A}$ l'orée de l'oeil paru la même année (cf. Figure II). Le dessin ne porte plus le

11. C.f. Schefer, Jean-Louis, Scénographie d'un tableau, Paris, Éditions du Seuil, 1969, pp. 120-138.

12.Id., p. 136

13. Lyotard, Jean-François, Discours, Figure, Paris, Editions Klincksieck, 1978, p. 271.

14. Eco, Umberto, op. cit., p. 147. 


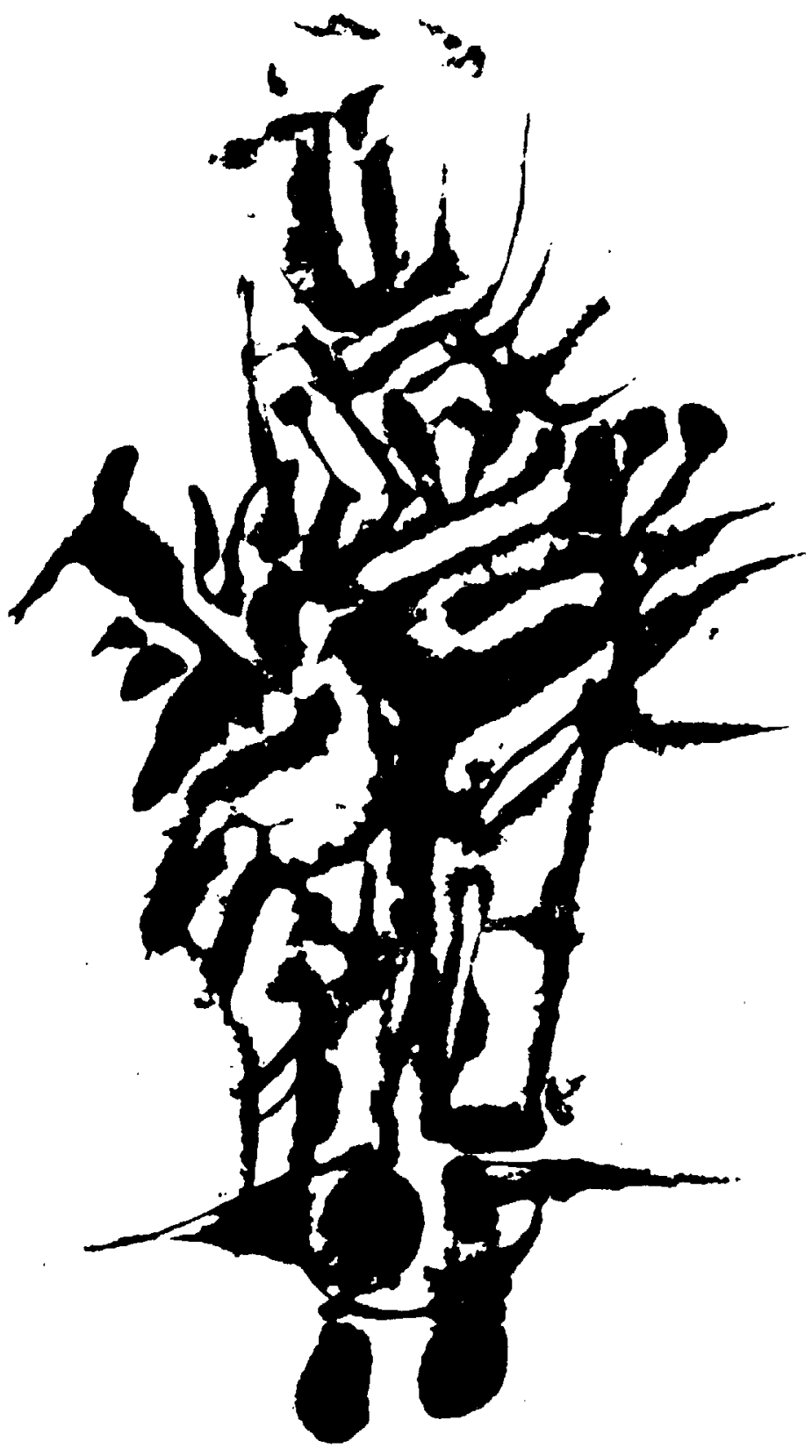

Figure II: «Portrait de Miror», extrait de $\dot{A}$ l'orée de l'oeil, éditions du Noroît. 
texte dénominatif qui accompagnait le Miror de 1953; seule, en bas, à gauche, l'inscription «Portrait de Miror» et la signature de l'artiste, à droite, scellent la feuille.

La rencontre de l'oeuvre graphique et du nom propre sur la même surface «qui fonctionne simultanément comme page d'écriture et support matériel, provoque un double effet critique, sur la figure, comme mimésis parfaite, sur le nom (propre) comme désignation totale; effet critique double où l'on reconnaîtra la circularité du nom et de l'image, de l'écriture et de la figure». ${ }^{15}$ Mais qu'en est-il du Miror dont on nous suggère le portrait?

Le Miror de 1953 portait l'équivocité de sa dénomination puisque la correspondance des textes iconique et verbal se répondait mutuellement en multipliant les effets de sens. Mais le Miror de 1957 offre un tout autre système opérationnel. D'abord le titre: il marque l'ouverture expansive d'un écart infinitésimal avec le continuum expressif et renvoie, non pas à luimême mais à ce qu'il clôt. Lorsque je lis "Portrait de Miror", ces mots font signe vers le dessin, deviennent en quelque façon lettres gestes qui pointent vers le même espace qu'elles occupent, mais un espace occupé par ce qu'elles montrent. Cette fonction métonymique se vide de son signifié puisque, devenu symbole indiciel, elle ne montre pas un ensemble graphique comme référence, mais elle désigne une feuille portant à la fois un dessin et son identification, soit elle-même. Il existe une bi-univocité des termes coexistants dans le même espace; le titre - signes gestuels - et l'oeuvre picturale; chacune de ces deux fonctions opérant en vertu d'un commensalisme qui approfondit la spécularité dù signifiant. Le processus d'identification est rendu possible parce que le nom «Miror» a, pour de nombreux contemporains de l'oeuvre première, des résonances mnémoniques grâce auxquelles s'intensifie le code de reconnaissance. Même si ce nom n'a pas de modèle premier, il y a une assimilation du portrait et du nom dans cette circularité du code qui fonctionne à vide puisque l'inscription est le support de l'être: déictique pur où le nom propre trouve son essence.

Alors que l'art du portrait était réservé traditionnellement à l'ostentation d'une puissance et la définition d'un Moi, dans le «Portrait de Miror», ce double rôle est très fortement atténué puisque l'individu premier, le Miror réel, n'existe pas. - Il importe de souligner que, de toute façon, le portrait n'est qu'une approximation d'un être (comment rendre le modelé de la peau, la finesse de l'épiderme, la complexité de l'oeil, etc.?). La parfaite mimésis est impossible à réaliser bien que, au niveau de la réplicabilité du réel, on peut parvenir, lorsqu'on opère au degré extrême des "échelles d'iconicité" telles que proposées par Abraham Moles, 16 à produire un ensemble de signes iconiques qui ne "possèdent pas les mêmes propriétés physiques que l'objet, mais qui mettent en oeuvre une structure perceptive

15. Marin, Louis, op. cit., p. 79.

16. Moles, Abraham, cité par Umberto Éco, op. cit., 1978, p. 146. 
«semblable» à celle que déclenche l'objet»:17 Le portrait peint peut, dans la conscience commune, être l'ersatz du prototype. - Dans le Miror première manière, le texte était en quelque sorte ancré à l'image, mis en procès représentatif, déterminé par la nature dénominative de la strophe renvoyant à la morphologie du personnage et à ses attributions. Le dessin de 1957 est de tout autre nature puisqu'il ne comporte qu'une indication minimale sur laquelle se fonde la lexie ultérieure. Également, dans le cas de Miror, nous sommes continuellement référés aux XXXI strophes du texte originaire sans que nous puissions déceler un seul phénomène de congruence. Impossibilité donc de saisir une quelconque ressemblance qui justifierait, même de façon arbitraire, l'appellation du nom propre. Reste le texte qui accompagne la première version graphique de Miror. Pouvons-nous émettre un jugement de «similarité» à partir «de critères de pertinence fixés par des conventions culturelles»? 18

L'oeuvre graphique de 1957 pourrait, à la rigueur, se fonder sur les références «iconographiques» contenues dans les cinq vers du dessin de 1953. Ainsi, à refaire une scénographie comme celle que nous avons effectuée précédemment, nous parviendrions sensiblement aux mêmes étapes démonstratives, à localiser les mêmes traits pertinents (poitrine trouée, corps déchiré, lacéré, ailes). Sauf que, dans ce cas-ci, le code iconique, défini selon Éco comme «un système qui fait correspondre à un système de moyens graphiques des unités perceptives et des unités culturelles codifiées, ou des unités pertinentes d'un système sémantique qui résulte d'une codification préalable de l'expérience perceptive», 19 se manifeste avec une plus grande complexité dans la mesure où la référence représentative est prise en charge par le rapport désignatum/définitum et s'y annule. Le dessin, exécuté à l'encre diluée, n'a pas la même faculté de représentation que celui de 1953 .

En peinture, je pars souvent d'une tache, d'un hasard provoqué, peu à peu se dessine une image qui veut apparaître et dès que je vois, je m'applique à faire émerger cette image (l'image «qui cogne à la vitre»), à la rendre aussi claire que possible. Ce qui n'était que virtuel devient visible. 20

Giguère part d'un noyau autour duquel s'élabore un tissu cellulaire hétérogène qui détermine la fígure. Les contours, souvent flous, indéterminés, circonscrivent des lieux anatomiques déduits plutôt que nettement énoncés. En suspens dans l'espace de la feuille, sans mise en scène, Miror est présenté sans principe de justification, ne serait-ce que par ce renvoi circulaire du nom et du portrait: tension interne «entre le jeu aux surfaces de

17. Eco, Umberto, op. cit., 1978, p. 153

18. Ibid., p. 154.

19. Ibid., p. 161.

20. Giguère, Roland, “À propos de...", op. cit., p. 166. 
l'être et l'englobement immédiat dans la «sphéricité» de la nomination».21

Il nous est possible d'appliquer le texte verbal de 1953 sur le continuum expressif de 1957 et d'en dégager une signification mais cette opération porte atteinte au procès autonymique qui se joue dans l'instance iconique. C'est dans le croisement des deux axes, le titre et l'image, que se noue la totalité des sens; même si le recours au cartouche du Miror original constitue une lexie possible, elle ne peut en aucun cas être exclusive et commande de sérieuses réserves quant à la motivation des signes puisque ceux-ci déclineraient la modalité de la stimulation; producteur «d'une espèce d'impression opérée par l'objet lui-même sur le continuum matériel de l'expression».22

C'est au niveau du discours verbal immédiat, en l'occurrence le titre, que nous pouvons pousser notre extrapolation. Nous avons montré précédemment que «la figuration propose un système d'expression et un système de signification ${ }^{23}$ dont le texte linguistique est le tissu conjonctif, le versant nominal de la figure. Cette spécificité lie la représentation iconique et la textualité qui en émerge forçant la lecture et l'astreignant à une mise en actes langagiers. De la même façon, le titre soumet le regard à une contrainte nominale et nous induit à rechercher une forme «humaine», là où la culture a enregistré sa présence, puisque le portrait s'applique à immortaliser des personnes, réelles ou fictives. À l'anthropomorphisme recherché dans le dessin, que suggère fortement le titre, seules quelques équivalences iconiques de la perception (tête, pieds, morphologie médiane) provoquent la reconnaissance du personnage qui, pourtant, n'a aucune matière du contenu. ${ }^{24}$ Comme nous l'avons déjà souligné, cette matière du contenu ne peut exister préalablement; elle naît avec la matière de l'expression et y repousse toute saisie du réel par le jeu de la représentation. Le chassé-croisé de la matière du contenu avec celle de l'expression structure un processus anamorphique indissociable du système des lexies sous-jacentes. Dès lors, le continuum expressif et l'indicateur verbal sont un creuset spéculaire; le signifiant de l'un devenant le signifié de l'autre et vice-versa, où le type du contenu, à cause de sa complexité et de sa motilité, échappe à l'identification.

Nous sommes en présence d'un corps figuré (le dessin proprement dit) et d'un corps signifié (l'ensemble titre-dessin-feuille entière) dont l'interaction avalise tout processus de signifiance par lequel il y a un surcodage de la figure. En somme la lexie, obligée de lexicaliser le dessin, surcharge l'espace du discours produit par le circuit de la connotation des figures/zones figuratives. La résistance de la figure au signifié analogique s'amoindrit dès

21. Marin, Louis, op. cit., p. 78.

22. Eco, Umberto, op. cit., 1978, p. 150.

23. Schefer, Jean-Louis, op. cit., p. 67.

24. C.f. Eco, Umberto, op. cit., 1978, p. 141. 
que le système de l'énonciation s'immisce dans le procès autonymique et en accentue la bi-univocité. Le «Portrait de Miror» (1957) atteint son maximum d'efficacité dans le scellement du titre et du dessin par l'inscription de la «métaphore métonymique» du portrait dont il extrait en quelque sorte sa prégnance.

On note, dans l'économie du système de figuration de ces deux dessins, que le continuum expressif tend à s'indexer au texte - strophe ou titre - comme lieu axiologique. Mais une telle auto-réflexivité suscite un va-et-vient continuel entre deux désignata permutables, écriture et dessin, en position instable: l'autre terme est à la fois son référent et son signifié. Cette situation se radicalise dans l'exécution graphique de 1957 puisque l'homologation, ou la circulation de l'identité, est circonscrite à la feuille elle-même. La métaphore métonymique prend en charge le code figuratif à partir duquel se crée un espacement entre les deux ordres non permutables: la lexie (l'opération de la lecture du dessin) et le titre. D'abord afférent, ce dernier suscite une stratification des discours car dessin et texte proxémique intériorisent l'un et l'autre le référent, d'où une représentation à double entrée. Le signifiant, imaginaire, sollicite une matière du contenu qui ne peut s'atteindre dans les lexies et le texte qu'au travers d'espaces communs aux deux (en les traversant) où se décomptent les métamorphoses du signe. 


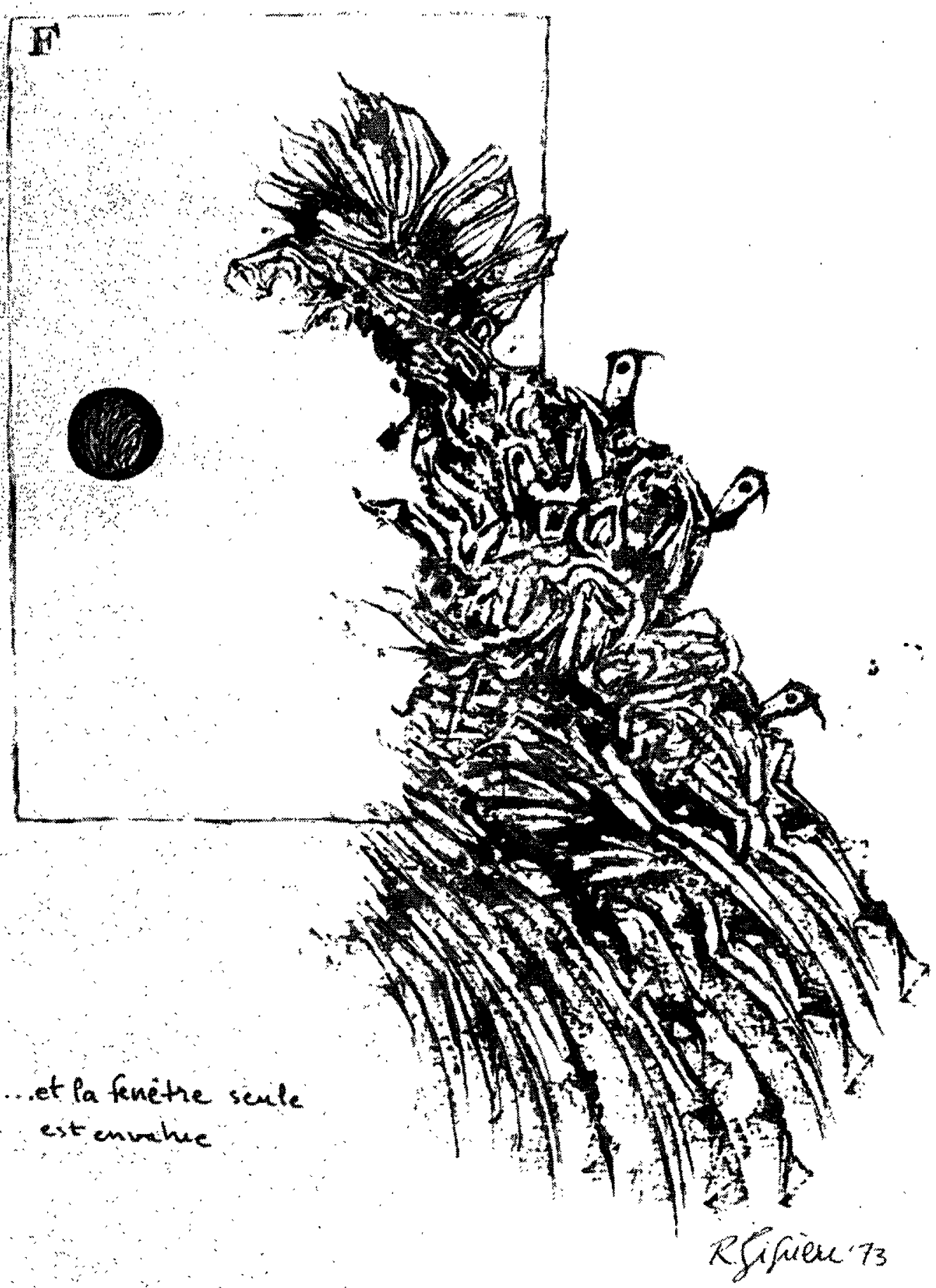

